\title{
Analysis of Y Chromosome Microdeletion and Karyotype in 516 Patients With Azoospermia
}

\section{Lihua Wu}

Guangdong Women and Children Hospital

Jingjing $\mathrm{Hu}$

Guangdong Women and Children Hospital

\section{Yiqun He}

Guangdong Women and Children Hospital

\section{Yicong Zhang}

Guangdong Women and Children Hospital

Kailing Liang

Guangdong Women and Children Hospital

\section{Li Guo}

Guangdong Women and Children Hospital

\section{Hanbiao Chen}

Guangdong Women and Children Hospital

Ting Wang ( $\nabla$ kevinwtwt@163.com )

https://orcid.org/0000-0002-9261-9016

\section{Research}

Keywords: Azoospermia, karyotype analysis, Y chromosome microdeletion

Posted Date: July 23rd, 2020

DOI: https://doi.org/10.21203/rs.3.rs-46015/v1

License: (c) (1) This work is licensed under a Creative Commons Attribution 4.0 International License.

Read Full License 


\section{Abstract}

Background: To investigate the correlation between Y chromosome microdeletion and cytogenetic analysis in patients with azoospermia.

Methods: A total of 516 patients with azoospermia were enrolled from March 2015 to December 2019. Karyotype analysis(G-banding) was performed with peripheral blood.Y-chromosome azoospermia factor (AZF) were detected by quantitative fluorescent polymerase chain reaction (QF-PCR).

Results: Chromosome abnormality was detected in 66 of the 516 azoospermia patients, with an abnormal rate of $12.8 \%$ In addition, $7.8 \%$ cases(40/516) presented with Y-chromosome AZF microdeletions, in which 27 cases patients with karyotype of $46, X Y(67.5 \%, 27 / 40)$ and 13 cases of chromosome abnormalities $(32.5 \%, 13 / 40)$.

Conclusion: The incidence of $Y$ chromosome microdeletion was higher in the patients with karyotype of $46, X Y$. Conventional cytogenetics cannot directly reflect the microdeletions of $Y$ chromosome. Therefore, the combination of this two detection methods can help to clarify the genetic causes of patients with azoospermia and provide a theoretical basis for clinical assisted reproductive technology.

\section{Background}

About $10-15 \%$ of couples with childbearing age worldwide are infertile, and the World Health Organization(WHO) considers that $50 \%$ of infertile couples are caused by male factors[1]. Besides, The azoospermia and occult azoospermia account for 10-20\% in male infertility. Several factors have been implicated in male with azoospermia such as genetic abnormality, abnormal sex hormone level, abnormal immunity, age, environment and others. The main cause of genetic factor involved in male with azoospermia, which was the most concerned by clinic, were chromosome abnormality and $Y$ chromosome microdeletion. Microdeletion in the AZF region of the $Y$ chromosome controlling spermatogenesis located in the q11 region of $Y$ chromosome. These aetiological examination is helpful for clinical treatment to assisted reproductive technology to reduce the economic burden of patients. In the present study, the frequency and type of major chromosomal abnormalities including $Y$ chromosome microdeletions of 516 patients with azoospermia were investigated, which is to help identify the genetic etiology of male azoospermia.

\section{Methods}

\section{Subjects:}

A total of 516 patients diagnosed as azoospermia were collected in the reproductive health and infertility department in our hospital from March 2015 to December 2019.The age ranged from 21 to 52, with an average age of 31.7. All the patients were examined by physical examination and semen routine analysis to rule out varicocele, endocrine disorders, genital inflammation, immune abnormalities, physical and 
chemical factors causing sperm loss. The diagnostic criteria for azoospermia are in accordance with the definitions and methods of WHO's 5th edition of the "Human Sperm Examination and Handling Laboratory Manual" [2]. The sperm density is checked at least 3 times or more, and no sperm was seen after centrifugation.This study was approved by the Ethics Committee of Guangdong Women and Children Hospital

\section{Cytogenetic analysis}

Informed consent was taken from the patients and donors prior to collection of $4 \mathrm{ml}$ heparinised blood samples. Chromosome investigations were performed on cultures of peripheral blood lymphocytes using standard techniques. Karyotype analysis using GTG-banding was performed following standard procedures, At least 5 metaphases were analysed and 20 karyotypes were counted for each patient and increased up to more than 30 to 100 metaphases in case of mosaicism to confirm the result. the karyotypes were reported in accordance with the up-to-date International System for Human Cytogenomics Nomenclature 2016 (ISCN2016) [3].

\section{Y chromosome microdeletion analysis}

Genomic DNA was obtained from EDTA blood samples extracted from patients. Six sequence tagged sites (STS) in the AZF region were used as markers for analysis of microdeletion. The STS markers were the zinc finger $Y$-chromosomal gene and the sex-determining region of the $Y$ chromosome(SRY) geneincluded asinternal control (IC). PCR primers were labelled by FAM, JOE and TAMRA fluorescently. With the exception of the ZFX/ZFY primer, other primers and amplification conditions were carried out in accordance with the European reproductive society (EAA) 2004 guidelines and EMQN standards [4]. Finally, the results of capillary electrophoresis were analyzed by GeneMaker (version2.2.0) software.

\section{Results}

\section{Cytogenetic anlysis}

A total of 66 chromosome abnormalities were detected in 516 azoospermia patients, with a detection rate of $12.8 \%(66 / 516)$.Of these ,fifty-seven $(86.36 \%, 57 / 66)$ sex chromosome abnormalities were detected, including four cases with sex chromosome structural abnormalities, 44 sex chromosomal aneuploidies(SCA), four cases with disorders of sex development(DSD), and 9 cases with mosaicism .In addition, 9 cases $(13.64 \%, 9 / 66)$ of autosomal structure rearrangement were also detected as shown in Table 1.

\section{Y chromosome microdeletion}

AZF deletion was detected in 40 of the 516 patients diagnosed with azospermia, with a total deletion rate of 7.8\%(40/516), including $5 \mathrm{AZFa}(12.5 \%, 5 / 40), 1 \mathrm{AZFb}(2.5 \%, 1 / 40), 20 \mathrm{AZFc}(50.0 \%, 20 / 40), 9$ $A Z F b+A Z F c(22.5 \%, 9 / 40)$, and $4 A Z F a+A Z F b+A Z F c(10.0 \%, 4 / 40)$ as shown in Table 1. 
Table 1

Summary of $\mathrm{Y}$ chromosome microdeletion and karyotype in 516 patients with azoospermia

\begin{tabular}{|c|c|c|c|c|c|c|c|c|}
\hline \multirow[t]{2}{*}{ Type } & \multirow[t]{2}{*}{ karyotype } & \multirow{2}{*}{$\begin{array}{l}\text { Case(Abnormal } \\
\text { ratio \%) }\end{array}$} & \multicolumn{5}{|c|}{ AZF microdeletions } & \multirow{2}{*}{$\begin{array}{l}\text { Loss } \\
\text { ratio } \\
(\%)\end{array}$} \\
\hline & & & a & b & c & bc & $a b c$ & \\
\hline $\begin{array}{l}\text { Nomal } \\
\text { karyotype }\end{array}$ & $46, X Y$ & $450(87.2)$ & 5 & 1 & 19 & 2 & - & 67.5 \\
\hline \multirow{20}{*}{$\begin{array}{l}\text { Abnormal } \\
\text { karyotype }\end{array}$} & $47, X X Y$ & $39(7.56)$ & - & - & - & - & - & 0 \\
\hline & $46, X X$ & $4(0.78)$ & - & - & - & - & 4 & 10 \\
\hline & $\operatorname{mos} 47, \mathrm{XXY} / 46, \mathrm{XY}$ & $2(0.39)$ & - & - & - & - & - & 0 \\
\hline & $46, X, \operatorname{del}(Y)(q 11.22)$ & $4(0.78)$ & - & - & 1 & 2 & 1 & 10 \\
\hline & $47, X Y Y$ & $1(0.19)$ & - & - & - & - & - & 0 \\
\hline & $\operatorname{mos} 45, X / 46, X Y$ & $2(0.39)$ & - & - & - & 1 & - & 2.5 \\
\hline & $\operatorname{mos} 45, X / 46, X, Y q h-$ & $2(0.39)$ & - & - & - & 1 & - & 2.5 \\
\hline & $\begin{array}{l}\operatorname{mos} 46, X, \operatorname{del}(Y) \\
(q 11.22 q 11.23) / 45, X\end{array}$ & $1(0.19)$ & - & - & - & 1 & - & 2.5 \\
\hline & mos & $1(0.19)$ & - & - & - & 1 & - & 2.5 \\
\hline & $\begin{array}{l}\text { 46,X, } \operatorname{del}(Y) \\
(q 11.22) / 46, X, \operatorname{idic}(Y)\end{array}$ & & & & & & & \\
\hline & $(\mathrm{q} 11.22) / 45, \mathrm{X} / 46, \mathrm{XY}$ & & & & & & & \\
\hline & $\begin{array}{l}\operatorname{mos} 45, \mathrm{X} / 46, \mathrm{X}, \operatorname{der}(\mathrm{Y} ; \mathrm{Y}) \mathrm{t}(\mathrm{q} ? \\
\text { 11.23;q11.2)del(Y)(p?11.1) }\end{array}$ & $1(0.19)$ & - & - & - & 1 & - & 2.5 \\
\hline & $46, \mathrm{XY}, \mathrm{t}(18 ; 22)(\mathrm{p} 11.2 ; \mathrm{q} 11.1)$ & $1(0.19)$ & - & - & - & - & - & 0 \\
\hline & $46, X Y, t(1 ; 22)(q 12 ; p 12), 14 p+$ & $1(0.19)$ & - & - & - & - & - & 0 \\
\hline & $46, X Y, t(2 ; 5)(q 13 ; q 13)$ & $1(0.19)$ & - & - & - & - & - & 0 \\
\hline & 45,XY,der(13;14)(q10;q10) & $2(0.39)$ & - & - & - & - & - & 0 \\
\hline & $\begin{array}{l}46, \mathrm{XY}, \operatorname{der}(1) ? \operatorname{inv}(1)(\mathrm{p} 36.2 \mathrm{q} 21) ? \\
\text { inv(1)(p31q36.1) }\end{array}$ & $1(0.19)$ & - & - & - & - & - & 0 \\
\hline & 46,XY,inv(1)(p33q44) & $1(0.19)$ & - & - & - & - & - & 0 \\
\hline & 46,XY,inv(15)(q11.2q24) & $1(0.19)$ & - & - & - & - & - & 0 \\
\hline & 46,XY,inv(1)(q34.2q24) & $1(0.19)$ & - & - & - & - & - & 0 \\
\hline
\end{tabular}




\begin{tabular}{|c|c|c|c|c|c|c|c|c|}
\hline \multirow[t]{2}{*}{ Type } & \multirow[t]{2}{*}{ karyotype } & \multirow{2}{*}{$\begin{array}{l}\text { Case(Abnormal } \\
\text { ratio \%) }\end{array}$} & \multicolumn{5}{|c|}{ AZF microdeletions } & \multirow{2}{*}{$\begin{array}{l}\text { Loss } \\
\text { ratio } \\
(\%)\end{array}$} \\
\hline & & & a & b & c & bc & $a b c$ & \\
\hline Total & & $516(100)$ & 5 & 1 & 20 & 9 & 5 & 100 \\
\hline
\end{tabular}

\section{Discussion}

Chromosome abnormality and $\mathrm{Y}$ chromosome microdeletion are two genetic factors that most common caused in patients with azoospermia[5].Deletions involving the three AZF regions on Yq11 have been verified to affect spermatogenesis and to cause infertility. In this study, the detection rate of chromosomal abnormalities in 516 patients with azoospermia was $12.8 \%$, which is similar with the litreature 14.71\%(Li Fu et al, 2012)[6]. We found that the most frequent chromosome aberration was $47, X X Y$ in patients with azoospermia, accounting for $49.4 \%$ of the chromosomal abnormalities, which was consistent with the $51.14 \%$ reported by M. Zhang [7]. Klinefelter syndrome (KS) refer to congenital testicular seminiferous tubule hypoplasia. The classic karyotype for men with KS is $47, \mathrm{XXY}$. The SRY gene, which controls testicular development with male phenotype, is present on the $Y$ chromosome. However, $Y$ chromosome function is inhibited due to the extra $X$ chromosome, affected the development of testicular convoluted tubules by hyaline degeneration and fibrosis of spermatogenic tubules. The clinical manifestations of KS were spermatozoa, small and hard testicles, and dysplasia of secondary sexual characteristics. Neither AZF microdeletion of $Y$ chromosome was detected in 165 cases of $47, \mathrm{XXY}$ in patients with azoospermia or occult azoospermia reported by Liu[7],nor in our report. It was suggested that there was no significant correlation between the etiology of azoospermia and $\mathrm{Y}$ chromosome microdeletion in patients with KS, which indicates that may be there is no necessary for detection of AZF microdeletions may in patients with KS.

There are more than 2,000 genes on the autosomes that are involved in spermatogenesis from domestic and foreign research. Several studies have found genes associated with spermatogenic function on chromosome 1, including scp-1,tsMCAK,MTHFR,MSH4, and mmp-23 [8-9]. In this paper, there were 9 cases of autosomal abnormalities were be found in 516 patients with azoospermia, among which chromosome 1 was the most common, accounting for $44.4 \%$ of autosomal abnormalities, including 3 cases of inversion and 1 case of balanced translocation of chromosome 1, none of which had AZF microdeletion. Previously published literature showed that the number of broken sites on chromosome 1 is higher than other autosomes in male infertility, especially in azoospermatism patients[10]. All of these findings demonstrated that structural abnormalities of chromosome 1 may be highly correlated with azoospermatism patients. After the translocation or inversion of chromosome 1, if these broken sites are located in the genes involved in the formation of spermatogenesis, the integrity of the gene is destroyed, 
affecting gene expression and causing sperm disorder. Its mechanism remains to be further research in the future.

In our study, we found that 40 out of 516 patients with azoospermia suffered from the AZF microdeletion of $Y$ chromosome, with a total frequency of $7.8 \%$, be similar to what Ferlin [11] has reported. The three regions of AZF that can be fruency missing are named as AZFa, AZFb, and AZFc. They are located at the near, middle and far ends of Yql1.23. AZFa microdeletion can lead to sertoli cell only syndrome, which is characterized by decreased of mesenchymal cells and Sertoli cell through clinical pathologic examination, with the dysplasia of seminal convoluted tubules. And AZFb microdeletion can result in the Maturation and growth of spermatogoniums and primary spermatocytes, whose symptoms are azoospermia. However AZFc microdeletion is so much complex that can result in from a normal phenotype sperm to azoospermia. It was found that half of males with AZF microdeletion of $Y$ chromosome were AZFc microdeletions, which was the most common deletion type, followed by AZFb + $\mathrm{AZFc}(22.5 \%), \mathrm{AZFa}(12.5 \%), \mathrm{AZFa}+\mathrm{AZFb}+\mathrm{AZFc}(10.0 \%)$, and $\mathrm{AZFb}(2.5 \%)$. The inability to produce sperm associated with the loss of one or more regions of AZFa and AZFb. Zoospermatism patients with AZFC microdeletions of $Y$ chromosome can obtain mature sperm by testicular puncture, and the progeny can be injected by intracytoplasmic sperm injection (ICSI)[12-13].However, AZFc microdeletions still risk vertical transmission to male offspring. Therefore, to detect the regions of AZF microdeletions of patients with azoospermia provides a scientific basis for the choice of ICSI assisted fertility therapy in the future.By the way, genetic counseling was provided for the selection of female embryos by genetic diagnosis before ICSI treatment for patients with AZFc microdeletions, which reduced the unnecessary invasive surgery and reduced the psychological pressure and financial burden of patients.

The main genes that determines the development of spermatogenic cells in the testicular convoluted spermatogenic duct is located on q11 of $\mathrm{Y}$ chromosome, and this partial deletion or rearrangement may result in the deletion or alteration of a series of genes related to spermatogenic function on $Y$ chromosome. Among the 4 cases with 46, XY, del(Y) (q11.22) karyotypes we detected, we found a significant cytogenetic deletion of the long arm of the $Y$ chromosome, which involved AZF microdeletions in different regions, including 1 case of AZFc region deletion, 2 cases of AZFb $+A Z F c$ region deletion, and 1 case of AZFa + AZFb + AZFc region deletion. In addition, we detected AZF gene deletions in different regions and showed azoospermia in these $Y$ chromosome long arms with significant deletions. We also found that AZF was present at the position q11 on the $\mathrm{Y}$ chromosome. in our study we found that all of this 4 patients with AZF microdeletions was localized in q11.22 on Y chromosome, and the molecular level showed different AZF deletion. These results suggest that the possibility of AZF microdeletion was higher in patients with asthenia who are cytogenetically deficient in long arm q11.22 of $Y$ chromosome, and the missing region needs to be confirmed by molecular genetics.

In conclusion, chromosomal abnormalities and $\mathrm{Y}$ chromosome microdeletions are important genetic factors affected male with azoospermia. The majority of azoospermic patients with Y-chromosome AZF microdeletions have normal karyotypes. Conventional cytogenetics cannot directly reflect the situation of AZF microdeletion and must be clearly diagnosed by molecular genetics. The combined detection of 
these two methods helps to clarify the genetic causes of patients with azoospermia before receiving assisted reproduction treatment, thus reducing the psychological burden of patients, while avoiding unnecessary clinical treatment.

\section{Abbreviations}

AZF:azoospermia factor;QF-PCR:quantitative fluorescent polymerase chain reaction;WHO:World Health Organization;DSD:disorders of sex development;KS:Klinefelter syndrome;SRY:sex-determining region of the $Y$ chromosome;ICSI:intracytoplasmic sperm injection

\section{Declarations}

Acknowledgements:We would like to express our sincere gratitude to our patients and their family for their cooperation.

Authors'contributions:All authors have materially participated in the study and manuscript preparation.LW analyzed the clinic data, drafted the manuscript; JH and LG carried out the clinic data analysis, and participated in the design of the work; $\mathrm{KL}$ and $\mathrm{YH}$ participated in AZF analysis. $\mathrm{YZ}$ and $\mathrm{HC}$ participated in the karyotyping analysis.TW participated in the revision and design of the article.All authors have approved the final article.

\section{Funding}

Not applicable.

\section{Availability of data and materials}

The datasets used and/or analyzed during the current study are available from the corresponding author on reasonable request.

\section{Ethics approval and consent to participate}

This study was performed with the approval of Medical Ethics Committee of Guangdong Women and Children Hospital

\section{Consent for publication}

All patients in this report provided their consent for publication.

\section{Competing interests}

The authors declare that they have no competing interests.

\section{References}


1. World Health Organization.WHO manual for the standardized investigation diagnosis and management of the infertile male.Cambridge:Cambridge University Press,2005,27(3),100.

2. World Health Organization.WHO laboratory manual for the examination and processing of human semen[M].5thed.Geneva:World Health Organization,2010.

3. McGowan-Jordan J,Simons A,Schmid M.ISCN:an international system for human cytogenomic nomenclature (2016). Basel: Karger; 2013.

4. Navarro-Costa PWilkeMHoefsloot L,et al.The EAA/EMQN external quality control program critically improves the molecular diagnosis of $Y$ chromosome microdeletions.European Journal of Human Genetics,2019:167-168.

5. Abur U. Gunes S,Ascı R,etal. Chromosomal and Y-chromosome microdeletion analysis in 1,300 infertile males and the fertility outcome of patients with AZFc microdeletions. Andrologia. 2019;51(11):13402.

6. Li Fu,Da Ke Xiong,XianPing Ding.Genetic screening for chromosomal abnormalities and $Y$ chromosome microdeletions in Chinese infertile men.J Assist Reprod Genet,2012,29(6):521-52.

7. Zhang M,HT Fan,QS Zhang,etal.Genetic screening and evaluation for chromosomal abnormalities of infertile males in Jilin Province,China[J].Genetics and Molecular Research,201514(4):16178-84.

8. Paquis-Flucklinger V, Santucci-Darmanin S, Paul R, Saunieres A, Turc-Carel C,Desnuelle C.Cloning and expression analysis of ameiosis-specific Mut Shomolog:the Human MSH4 Gene.Genomics.1997,44(2):188-194.

9. Velasco G. Pendás AM,Fueyo A,etal.Cloning and characterization of human MMP-23,a new matrix metalloproteinase predominantly expressed in reproductive tissues and lacking conserved domains in other family members. The Journal Of Biological Chemistry. 1999;274(8):4570.

10. Bache I, Assche EV. Cingoz1 S,etal.An excess of chromosome 1 breakpoints in male infertility. Eur J Hum Genet. 2004;12:993-1000.

11. Ferlin A,Arredi B,SDeltra E,eta1.Molecular and clinical characterization of $Y$ chromosome microdeletions ininfertile men:a 10-year experience in Italy[J].JclinEndoc`nolMetab,2007,92(3):762770 .

12. OatesR D, Silber S. Brown LG,eta1.Clinical Characterization of 42 oligospermic or azoospermic men with microdelefon of AZFc region of the $\mathrm{Y}$ chromosome and of 18 children conceived via. ICSI[J]Human Reproduction. 2002;17:2813-24.

13. Park $\mathrm{SH}$, Lee HS, Choe $\mathrm{JH}$,et al. Success rate of microsurgical multiple testicular sperm extraction and sperm presence in the ejaculate in korean men with y chromosome microdeletions.[. J]Korean $\mathrm{J}$ Urol. 2013;54(8):536-40. 